\title{
Research on Applications of Graphene Composites in Electrochemical Detection
}

\author{
Xiaoyu Min ${ }^{1, a}$ \\ ${ }^{1}$ College of Materials and Chemical Engineering, Hainan University, Haikou, 570228, China
}

Keywords: Graphene composites, Electrochemical detection, Electrochemical sensor

\begin{abstract}
Graphene has rich and unique physical and chemical properties due to its single atomic layer structure. Graphene and its composites have been widely used in the field of materials science for a short period of ten years after the discovery. This paper will explore the preparation and applications in electrochemical detection of three kinds of graphene composite materials, including graphene/polyaniline, graphene/polypyrrole and graphene/ $\mathrm{MnO}_{2}$, to provide some references for the relative researchers.
\end{abstract}

\section{Introduction}

Graphene is a two-dimensional crystal. The common graphite is plane carbon atoms stacked by a layer with honeycomb ordered formed. The graphite layer interaction is weak, which is easy to form each other off, graphite sheet. When the graphite sheet is stripped into a single layer, a single layer of carbon atoms is graphene. The latest discovery of graphene is one of the most effective ways to prevent corrosion. Graphene is not only used in the electronics industry star, traditional industry prospects are limitless. Graphene is a two-dimensional carbon atom with SP2 hybrid orbitals, is the world's thinnest single atomic thickness of the material. Graphene has many remarkable characteristics. It has excellent electrical properties. The electron transfer rate can reach $200000 \mathrm{~cm}^{2} \mathrm{~V}^{-1} \mathrm{~S}^{-1}$ at room temperature. Graphene thermal conductivity is very good, thermal conductivity of $5000 \mathrm{Wm}^{-1} \mathrm{~K}^{-1}$. We introduce some functional groups or change the structure of graphene materials to obtain graphene composites. They have excellent physical and chemical properties, effectively expand and enhance the application value of graphene. This paper mainly discusses the preparation and electrochemical detection performance of grapheme/polyaniline composites, graphene/polypyrrole and graphene $/ \mathrm{MnO}_{2}$.

\section{Preparation and application of graphene/polyaniline in Electrochemical Detection}

Preparation. Dispersion blending method is the simplest method to prepare graphenepolyaniline electrode material. However, due to the poor solubility of conductive polymers such as polyaniline in many solvents, it is easy to aggregate and precipitate. We are through the vacuum dispersion properties, graphenepolyaniline films were prepared. The film in the specific capacitance of $210 \mathrm{~F} / \mathrm{g}$ is about 10 times of pure polyaniline film. In addition, due to the existence of graphene network, the swelling shrinkage behavior of polyaniline fibers in the circulation process is inhibited, so that the electrochemical stability of the super capacitor is greatly improved. First of all, on graphene suction with sodium polystyrene sulfonate negative charge, then the polyaniline nano fiber with positive charge adsorbed onto the graphene sheet, finally through the vacuum filtration method to prepare electrode materials. The materials of the specific capacitance with high specific surface area of electric double layer capacitance characteristics of the synergistic effect of graphene and larger capacitance between the polyaniline is the main reason for the electrochemical properties of materials can be improved. Recently, more and more attention has been paid to electrostatic adsorption. Firstly, polyaniline hollow spheres were prepared by electrostatic adsorption, and then, the negatively charged grapheme oxide wrapped in a hollow spherical surface with positive charge, and then gets the reduction of graphene by electrochemical reduction method. We prepare the electrode material with $614 \mathrm{~F} / \mathrm{g}$ capacitance, which only loses $10 \%$ after 500 capacitancecycles. 
Application. Figure 1 shows the cyclic voltammetric curve of the graphene/polyaniline composite material. The cyclic voltammetry curve figure close to a rectangle for cyclic voltammograms of graphene, indicating that it has good performance of electric double layer capacitor; and graphene/polyaniline composite cyclic voltammetry has obvious oxidation the reduction peak, 1 peaks corresponding to polyaniline reduced state generation, peak 1 is the corresponding reduction peak; peak 2 reaction generates intermediate oxidation states; peak 3 represents the complete oxidation of polyaniline generation, 3 peaks corresponding to its peak. This is the main source of pseudocapacitor. Cyclic voltammograms of composites and polyaniline is close to the peak position slightly offset, which may be the interaction results of graphene and polyaniline.

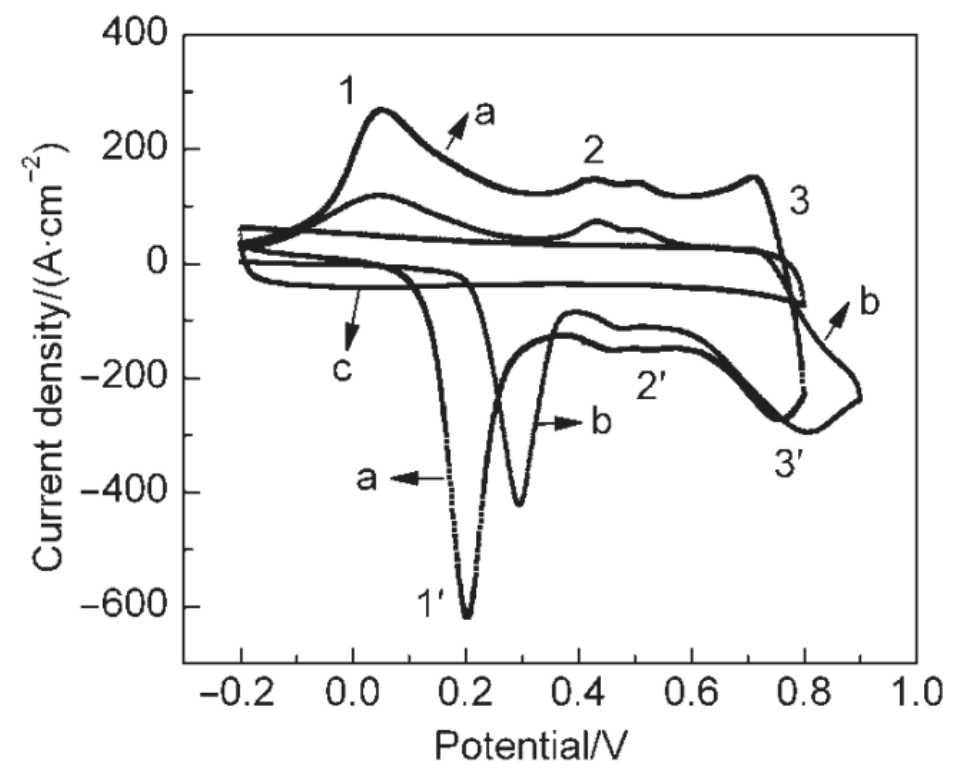

Fig.1: Cyclic voltammetry curve of graphene/polyaniline

\section{Preparation and Application of Graphene/Polypyrrole in Electrochemical Detection}

Preparation. The composite films on graphene surface can be prepared by depositing conductive polymers. This kind of graphene based composite films can be used as supercapacitor electrode, drive, sensor and other important materials, the performance is often better than their single material. Usually, it is used in situ electrochemical deposition of pyrrole monomer in graphene films on graphene /polypyrrole. In addition, to obtain more homogeneous coating by pulse electroplating technology can also be compared with continuous evaporation method, pulse electroplating technology can reduce the blocking and obtain more continuous microporous coating, the graphene surface of /polypyrrole film is more perfect. On the other hand, in addition to double-layer graphene /polypyrrole films, with more complex multilayer graphene films were prepared by polypyrrole, we can through electrostatic interaction between graphene oxide film will be negatively charged and positively charged surfactant micelle effects together, and then thepolymerization between graphene oxide layers, the graphene nano polypyrrole thin film sandwich structure the. Due to the lack of functional groups on the surface of graphene sheet, it is not easy to integrate with other materials. Therefore, the chemical modification of graphene surface is an important step to obtain various functional composites.

Application. As the electrochemical actuator, polypyrrole has already been studied for many years as the polypyrrole is lightweight and soft. The volume change potential advantages ofpolypyrrole stimulation mechanism based on the electrochemical oxidation reduction under the action of alternating state gained or lost. Unlike polypyrrole, graphene the stimulus response mechanism to drive the process of charge and discharge charge. Electron injection to the charge accumulated in the process of graphene will cause swelling, and the hole injection will shrink, the graphene layer between the polypyrrole and the driving mechanism of the difference, it is reasonable to design a new type of graphene/polypyrrole composite drive based on the material. As is shown in 
Figure 2, in the case of electrochemical actuation, the double layer of the actuator will swing around the voltage. The thin film of the graphene /polypyrrole bilayer can be swung around 0.8 Vand the curvature angle can reach 120.

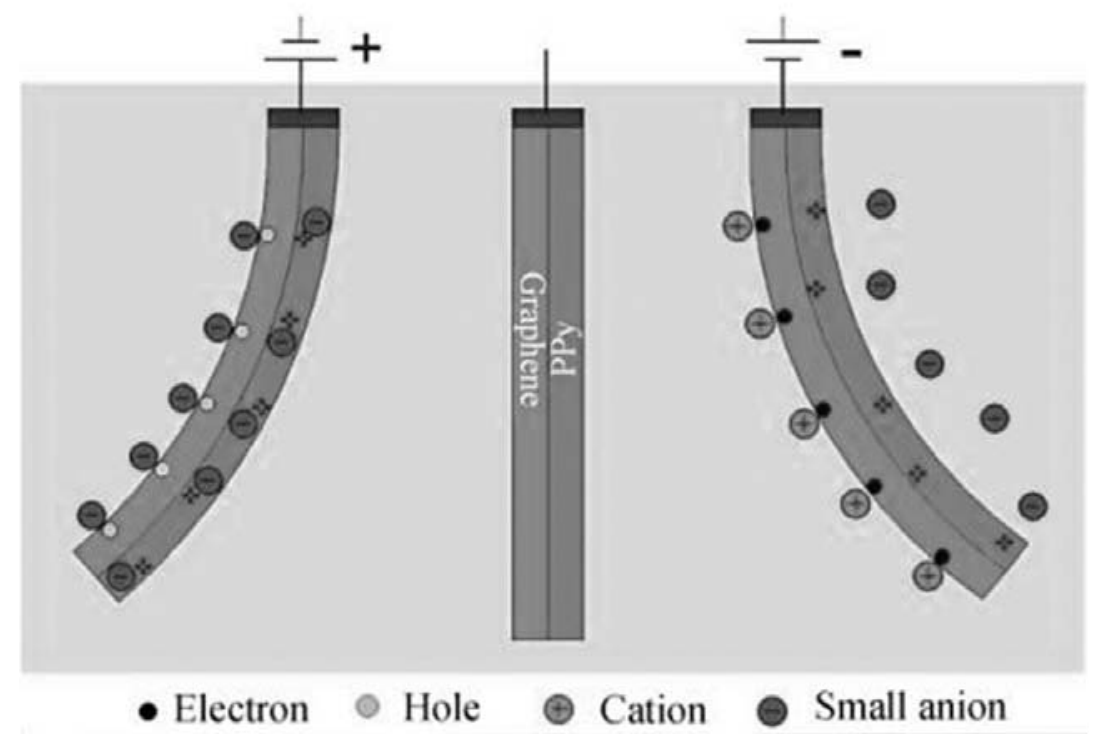

Fig. 2: Actuation mechanism of graphene/polypyrrole bilayer structure

\section{Preparation and Application of Graphene $/ \mathrm{MnO}_{2}$ in Electrochemical Detection}

Preparation. We use the preparation of graphene/MnO2 composites by co precipitation method. Compared with the reported preparation method, the experimental conditions of this method are simple and easy to operate. The composite of graphene and graphene by using manganese dioxide, large specific surface area, high conductivity and high manganese dioxide capacity, the manganese dioxide load on the surface of graphene, manganese dioxide increased the specific surface area and reduce the resistance of the composite materials, thereby improving the electrochemical performance. And through the preparation of different mass ratio of the composite material, discuss the influence of $\mathrm{MnO}_{2}$ proportion on the properties of the composite materials, and obtain the best ratio of manganese dioxide and graphene. The muffle furnace prepared GO into 1050 DEG C in pyrolysis 30s, after removal of dispersed in anhydrous ethanol with $100 \mathrm{~W}$ ultrasonic power $2 \mathrm{~h}$, filtering and washing drying after grinding, obtain graphene. The graphene was dispersed in deionized water, adding a certain amount of Potassium Permanganate, stirring slowly after manganese acetate dropping $0.01 \mathrm{~mol} / \mathrm{L}$. The potassium permanganate and manganese acetate molar ratio is $2: 3$. We drop after the completion of the reaction, using deionized water and anhydrous ethanol washing, filtering and drying to obtain manganese dioxide/ graphitegraphene composite materials. The reaction equation is: $2 \mathrm{MnO}_{4}^{-}+3 \mathrm{Mn}^{2+}+2 \mathrm{H}_{2} \mathrm{O}=5 \mathrm{MnO}_{2}+4 \mathrm{H}^{+}$

Application. The deposition amount of manganese dioxide more, but not fully covered the surface of graphene, graphene and electrolyte contact surface increased, can improve the performance of capacitor. Moreover, the particle size affects the specific surface area of the electrode material. Therefore, manganese dioxide too much, which leads to the formation of larger particles, the electrode surface area of the material is reduced, the electrode material and the electrolyte contact surface is reduced, resulting in the decrease of its electrochemical properties. It is shown in Figure 3, when the mass fraction of graphene is large, the standard curve of an isosceles triangle. It shows that the electrode material circulation has strong stability, charge discharge curve symmetry, reflects the good performance of the capacitor. The voltage is about $0.1 \mathrm{~V}$ parts of the platform due to the polarization effect in the electrolyte of manganese dioxide. 


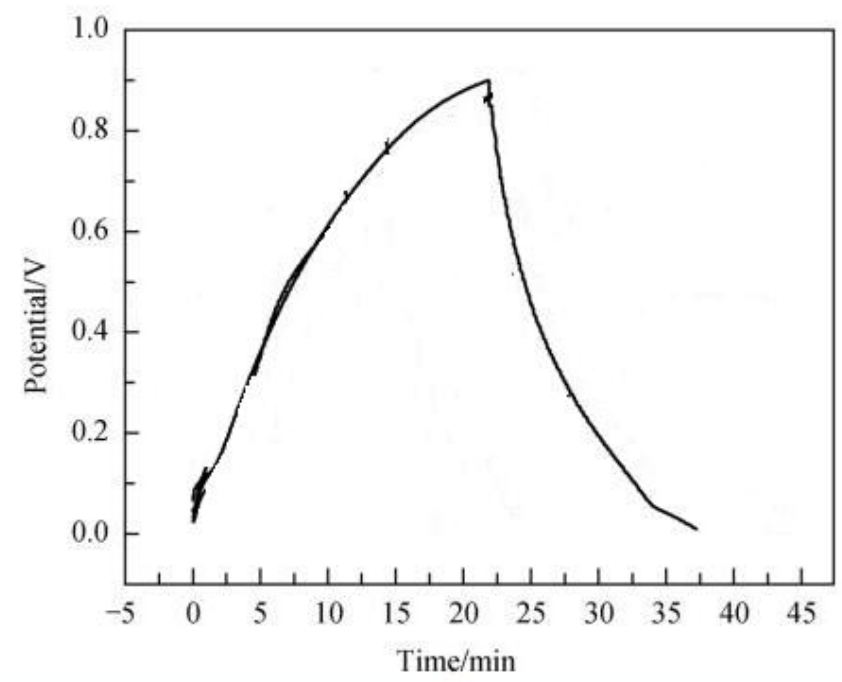

Fig 3: Current charge and discharge curves of graphence/ $\mathrm{MnO}_{2}$ composite

\section{Conclusion}

In this paper, we focus on the applications of graphene composite materials in electrochemical detection. The performances of three kinds of graphene composite materials in electrochemical detection are demonstrated. Of course, the electrochemical performance of graphene composites is still under exploration, which needs further broadening and deepening.

\section{References}

[1] Wang Hongzhi, GaoCuixia, Zhang Peng, Yao Suwei, Zhang Weiguo, Synthesis and Electrochemical Performance of Graphene/Polyaniline [J]. ActaPhysico-ChimicaSinica, 2013, 29(1): 117-122.

[2] Yingyue Zhang, AmmarAlgburi, Ning Wang, VladyslavKholodovych, Drym O. Oh, Michael Chikindas, Kathryn E. Uhrich, Self-assembled Cationic Amphiphiles as Antimicrobial Peptides Mimics: Role of Hydrophobicity [J]. Linkage Type, and Assembly State, Nanomedicine: Nanotechnology, Biology and Medicine, 2017, 13(2): 343-352.

[3] Song Yingpan, Feng Miao, Zhan Hongbing, Applications of Graphene Nanocomposites in Electrochemical Biosensors, Progress in Chemistry, 2012, 24(9): 1665-1673.

[4] Chen Nan, Hu Yue, Zhao Yang, QuLiangti, Progress in Controllable Preparation and Electrochemical Applications of Graphene/Poly (pyrrole) Composites [J]. Acta Polymerica Sinica, 2014 (6): 752-760.

[5] Dalia S. Abdelhamid, Yingyue Zhang, Daniel R. Lewis, Prabhas V. Moghe, William J. Welsh, Kathryn E. Uhrich, Tartaric Acid-based Amphiphilic Macromolecules with Ether Linkages Exhibit Enhanced Repression of Oxidized Low Density Lipoprotein Uptake [J]. Biomaterials, 2015(53): 32-39. 